\title{
Quantum-enhanced stimulated emission detection for label-free microscopy
}

Cite as: Appl. Phys. Lett. 117, 024002 (2020); https://doi.org/10.1063/5.0009681

Submitted: 02 April 2020 . Accepted: 19 June 2020 . Published Online: 13 July 2020

Gil Triginer Garces (D), Helen M. Chrzanowski, Shakib Daryanoosh, Valerian Thiel, Anna L. Marchant (D), Raj B. Patel (D), Peter C. Humphreys, Animesh Datta ${ }^{(D)}$, and lan A. Walmsley

\section{COLLECTIONS}

Paper published as part of the special topic on Quantum Sensing with Correlated Light Sources
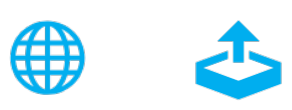

\section{ARTICLES YOU MAY BE INTERESTED IN}

Two-photon phase-sensing with single-photon detection

Applied Physics Letters 117, 024001 (2020); https://doi.org/10.1063/5.0009527

Axially controllable multiple orbital angular momentum beam generator Applied Physics Letters 117, 021101 (2020); https://doi.org/10.1063/5.0011445

Quantum dense metrology by an SU(2)-in-SU $(1,1)$ nested interferometer Applied Physics Letters 117, 024003 (2020); https://doi.org/10.1063/5.0012304

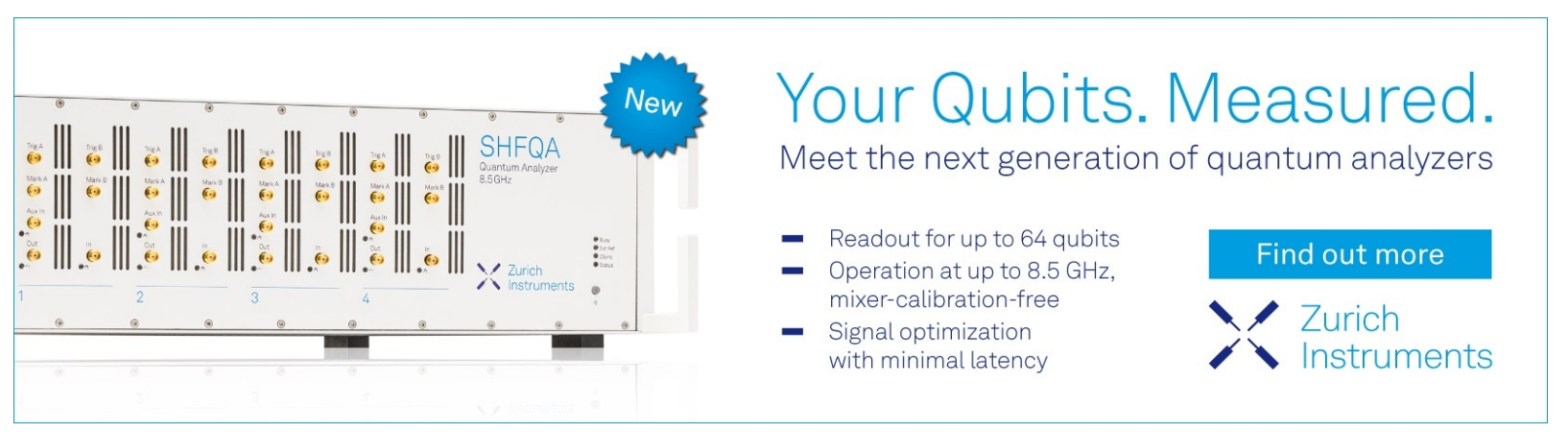




\title{
Quantum-enhanced stimulated emission detection for label-free microscopy
}

\author{
Cite as: Appl. Phys. Lett. 117, 024002 (2020); doi: 10.1063/5.0009681 \\ Submitted: 2 April 2020 - Accepted: 19 June 2020 . \\ Published Online: 13 July 2020
}

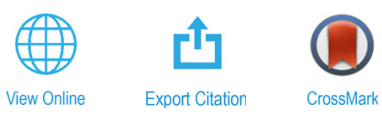

Gil Triginer Garces, ${ }^{1}$ (D) Helen M. Chrzanowski, ${ }^{1,2}$ Shakib Daryanoosh, ${ }^{7}$ Valerian Thiel, ${ }^{1,3}$ Anna L. Marchant, ${ }^{1}$ Raj B. Patel, ${ }^{1}$ (D) Peter C. Humphreys, ${ }^{1}$ Animesh Datta, ${ }^{4, a)}$ (D) and Ian A. Walmsley, ${ }^{7,5, b)}$

\author{
AFFILIATIONS \\ 'Department of Physics, University of Oxford, Clarendon Laboratory, Parks Road, Oxford OX1 3PU, United Kingdom \\ ${ }^{2}$ Humboldt University of Berlin, Unter den Linden 6, 10099 Berlin, Germany \\ ${ }^{3}$ University of Oregon, 120 Willamette Hall, Eugene, Oregon 97403, USA \\ ${ }^{4}$ Department of Physics, University of Warwick, Coventry CV4 7AL, United Kingdom \\ ${ }^{5}$ Imperial College London, South Kensington, London SW7 2AZ, United Kingdom
}

Note: This paper is part of the APL Special Collection on Quantum Sensing with Correlated Light Sources

a)Electronic mail: animesh.datta@warwick.ac.uk

b) Author to whom correspondence should be addressed: ian.walmsley@imperial.ac.uk

\begin{abstract}
Nonlinear optical microscopy techniques have emerged as a set of successful tools for biological imaging. Stimulated emission microscopy belongs to a small subset of pump-probe techniques, which can image non-fluorescent samples without requiring fluorescent labeling. However, its sensitivity has been shown to be ultimately limited by the quantum fluctuations in the probe beam. We propose and experimentally implement sub-shot-noise limited stimulated emission microscopy by preparing the probe pulse in an intensity-squeezed state. This technique paves the way for imaging delicate biological samples that have no detectable fluorescence with sensitivity beyond standard quantum fluctuations.
\end{abstract}

Published under license by AIP Publishing. https://doi.org/10.1063/5.0009681

Fluorescence is a backbone of optical microscopy of biological systems, underlying the spectacular advances in super-resolution microscopy over the past two decades. ${ }^{1-3}$ However, several important proteins, such as hemoglobin and cytochromes, cannot be detected by fluorescence because their spontaneous emission is dominated by rapid non-radiative decay. To overcome this challenge, many methods begin by labeling a sample of interest with fluorescent proteins. This allows for the imaging of the sample, although at the risk of interfering with its biochemical properties. To avoid this unwanted side effect, Min et al. ${ }^{4}$ developed stimulated emission microscopy (SEM), a nonlinear microscopy technique able to image chromophores with undetectable fluorescence. However, it was shown that the sensitivity of SEM is practically limited by the shot noise of the stimulation beam, which is reflected in the lowest detectable concentrations for a given light dose or acquisition time. The measurement sensitivity can be improved by increasing either or both of the latter, but at the cost of the fast photobleaching and formation of chemical radicals. ${ }^{5}$

Quantum physics has opened a new paradigm for optical sensing by leveraging non-classical correlations in either the probe light, the interaction with the sample, or the detection stage. The achievements of quantum metrology include demonstrations of imaging with sensitivity surpassing the shot-noise limit, ${ }^{6-8}$ enhanced resolution beyond the diffraction limit using quantum correlations, ${ }^{9}$ tracking of motion, such as the movement of lipid granules diffusing through cells, ${ }^{10}$ and improving the sensitivity of Raman scattering spectroscopy ${ }^{11}$ and microscopy.

Here, we propose to improve the sensitivity of SEM by using an intensity-squeezed coherent state of light ${ }^{13}$ in the stimulation beam. This optical state exhibits sub-Poissonian photon statistics, i.e., the variance of the photon flux is smaller than its mean. As a consequence of the reduced intensity fluctuations in the measurement signal, the small amplification induced by the sample can be resolved more precisely. We prepare an ultrafast intensity-squeezed probe, suitable for this nonlinear microscopy technique, via the deamplification of coherent seed pulses in a single-pass optical parametric amplifier (OPA). In addition, we perform the shot-noise-limited direct detection of the intensity-squeezed light and observe reduced quantum fluctuations in the SEM signal. 
Many chromophores have very short-lived excited states with much faster non-radiative decay rates than their spontaneous emission rates. As a result, their feeble fluorescence is overwhelmed by the background and detector dark counts. Nevertheless, an excited molecule can be stimulated down to the ground state by an incident light field with a frequency corresponding to the energy of a transition in the molecule, which results in the creation of a new coherent photon. SEM uses this optical amplification phenomenon as a contrast mechanism for imaging chromophores with undetectable fluorescence. ${ }^{4}$

In SEM, an excitation beam with intensity $I_{\mathrm{E}}$, incident on a collection of chromophores, promotes them to an excited state [level 1 in Fig. 1(a)]. Subsequently, a stimulation beam with a longer wavelength probes the sample, resulting in the emission of light in the mode of the impinging stimulation beam. As a consequence, optical amplification takes place [Fig. 1(b)]. Assuming that the intensity of the stimulation beam is $I_{S}$, the intensity of the generated stimulated emission, $\delta I_{S}$, is ${ }^{4} \delta I_{S}=I_{S} N_{2} \sigma_{s} / A \propto I_{S} I_{\mathrm{E}} N_{0} \sigma_{\mathrm{s}} \sigma_{\mathrm{a}} / A^{2}$, where $N_{k}$ is the population in the $k$ th energy level [Fig. 1(a)], $A$ represents the waist area of the beam, and $\sigma_{\mathrm{a}}$ and $\sigma_{\mathrm{s}}$ are the absorption $(0 \rightarrow 1$ transition $)$ and stimulation ( $2 \rightarrow 3$ transition) cross sections, respectively. Here, it is assumed that under a non-saturating regime of the dynamics, the population of the excited state satisfies $N_{2} \propto N_{0} I_{\mathrm{E}} \sigma_{\mathrm{a}}$. The goal of SEM is to detect the small variations in $G_{\mathrm{SE}}=\left(1+\delta I_{\mathrm{s}} / I_{\mathrm{s}}\right)$ as the beams are scanned across a sample, which provides an estimation of the number of molecules in the ground state, $N_{0}$. Given that the stimulated emission gain is proportional to the overlap of the excitation and stimulation fields, this method offers an intrinsic three-dimensional sectioning at the foci of the beams, as well as a finer spatial resolution than absorption imaging.

However, due to the weakness of the SEM signal, its measurement is hindered by the low-frequency technical noise of the laser and

(a)

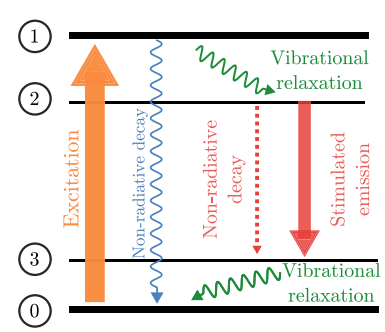

(c)

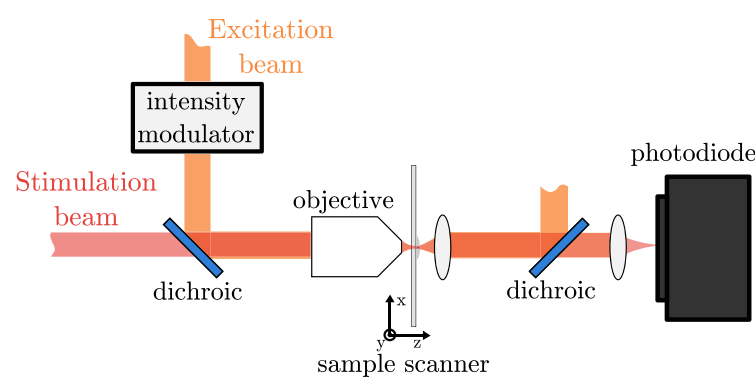

FIG. 1. (a) Energy level diagram for the stimulated emission-based pump-probe microscopy. ${ }^{4} 14$ (b) Amplification of the stimulation beam synchronized with the modulated excitation beam. electronics. In order to mitigate this technical noise, Min et al. ${ }^{4}$ implemented a modulation of the excitation beam at a few $\mathrm{MHz}$, such that the stimulation emission signal is moved to a sideband frequency beyond the technical noise in the system and can be separated using microwave filters. In this regime, the predominant limitation to the measurement of $G_{\mathrm{SE}}$ is the wideband shot noise in the stimulation beam. ${ }^{4}$

In a standard SEM experiment, the stimulation laser is well described as a train of ultrafast optical pulses in a coherent state. After traversing a sample together with a suitable excitation beam, and thereby picking up a time-dependent gain, these pulses are detected with a photodiode. In order to distinguish the small periodic amplification from the background noise, the resulting photocurrent is measured at the modulation frequency of the gain, e.g., by means of a spectrum analyzer (SA). We will now summarize the theoretical model of this measurement that we develop in the supplementary material (equations therein will be cited here with the prefix S), which suggests that the fundamental sensitivity limit set by the shot noise (that is, the quantum intensity fluctuations associated with the coherent states of light) can be overcome using squeezed light.

We model the optical gain in SEM as a phase-independent linear amplification mechanism and assume that the amplification noise can be ignored in the weak gain regime (S4). We allow the intensity gain to evolve in time according to $G_{\mathrm{SE}}(t)=G_{0}+\left(G_{0}-1\right) \cos \left(\Omega_{0} t\right)$, where the gain parameter $G_{0}$ is close to unity and $\Omega_{0}$ is an RF modulation frequency. As a result, the power spectral density (PSD) measured in the SA contains a narrowband component at $\Omega_{0}$. The amplitude of this signal is proportional to $\mathcal{S}_{\text {out }}\left(\Omega_{0}\right)=\left(G_{0}-1\right)^{2} P_{0}^{2} / 16$ [Eq. (S17)], where $P_{0}$ is the average optical power in the probe beam before the amplification process.

However, in addition to this narrowband signal, the measured PSD also contains noise of various origins. In the first place, the quantum fluctuations in the intensity of the probe field appear as a white (frequency-independent) noise contribution around the signal peak. According to a simple description of the optical quantum noise, and neglecting SEM amplification for the moment, the noise spectral density of quantum origin is $\mathcal{N}_{\text {in }}^{q}=\kappa P_{0} F$ [Eq. (S13)]. The constant $\kappa$ accounts for the particularities of the experimental implementation, and $F$ is analogous to the Fano factor of a random variable $F=1$ for a coherent beam (Poissonian statistics), and $F<1$ or $F>1$ for light fields with sub-Poissonian and super-Poissonian photon statistics, respectively. In the presence of the modulated SEM gain, the quantum noise spectral density is amplified to $\mathcal{N}_{\text {out }}^{q}=\mathcal{N}_{\text {in }}^{q} G_{0}$ [Eq. (S19)]. We consider a second source of noise: low-frequency intensity fluctuations of classical origin. ${ }^{15}$ In the absence of SEM amplification, the contribution of the classical technical noise, $\mathcal{N}_{\text {in }}^{t}(\Omega)$, is assumed to be non-zero only at frequencies well below $\Omega_{0}$. However, in the presence of gain, a fraction of this noise is modulated up in frequency, yielding a noise spectral density contribution $\mathcal{N}_{\text {out }}^{t}(\Omega)=\left(G_{0}-1\right)^{2} \mathcal{N}_{\text {in }}^{t}\left(\Omega+\Omega_{0}\right) / 16$ [Eq. (S20)].

The signal to noise ratio (SNR) of the SEM measurement, taken as the ratio between the signal and noise components of the measured $\mathrm{PDF}$ at the modulation frequency $\Omega_{0}$, is then [Eq. (S21)]

$$
\mathrm{SNR}=\frac{\mathcal{S}_{\text {out }}\left(\Omega_{0}\right)}{\mathcal{N}_{\text {out }}^{q}\left(\Omega_{0}\right)+\mathcal{N}_{\text {out }}^{t}\left(\Omega_{0}\right)}=\frac{\left(G_{0}-1\right)^{2} P_{0} / \kappa}{16 F G_{0}+\left(G_{0}-1\right)^{2} \rho_{t}},
$$


where we have defined $\rho_{t}=\mathcal{N}_{\text {in }}^{t}(0) /\left(P_{0} \kappa\right)$, which corresponds to the ratio between the spectral density of the low-frequency technical noise and the quantum shot noise in a coherent state $(F=1)$. It can be seen that, by increasing the power of the stimulation beam, $P_{0}$, the SNR improves but only at the risk of damaging the imaged sample. We, instead, propose to probe the sample with an intensity-squeezed coherent state of light. ${ }^{16}$ Such a state exhibits sub-Poissonian intensity statistics, i.e., $F<1$, yielding a higher $\mathrm{SNR}$ in the estimation of $G_{\mathrm{SE}}$ without increasing the intensity of the probe. While a lower $F$ immediately implies a greater SNR, it is worth noting that the enhancement will be largest when the technical noise is small compared to the quantum fluctuations. This condition is met in the low-gain regime that we are interested in, since technical noise contribution to the SNR is quadratic on $G_{0}$, while the quantum shot-noise contribution is linear.

A squeezed coherent state can be generated via the optical parametric amplification (OPA) of a seed coherent state. ${ }^{13}$ After an interaction with a suitable pump field, mediated by a nonlinear medium, the seed experiences a phase-sensitive amplification/deamplification, which renders its fluctuations quantum-correlated. We will here summarize the simple model of OPA developed in the supplementary material. Considering a pump and seed fields with average powers $P_{\mathrm{p}}$ and $P_{\mathrm{s}}^{\text {in }}$, respectively, the maximum/minimum powers of the amplified/deamplified seed, obtained for the corresponding extreme phase offsets between the seed and the pump, are [Eq. (S26)]

$$
P_{\mathrm{s}}^{\text {out }}=\left(1-\chi+\chi \exp \left( \pm \beta \sqrt{P_{p}}\right)\right) \eta P_{s}^{\text {in }},
$$

where $\beta$ is an effective nonlinearity, $\chi \in[0,1]$ is a coefficient that characterizes the mode overlap between the two interacting fields, and $\eta \in[0,1]$ is an efficiency that compounds the propagation loss and the detection efficiency.

In this situation, our model predicts the following maximum/ minimum intensity Fano factor for the amplified/deamplified seed [Eq. (S27)]:

$$
F \approx 1-\eta+\eta \frac{1-\chi+\chi \exp \left( \pm 4 \sqrt{P_{\mathrm{p}}} \beta\right)}{1-\chi+\chi \exp \left( \pm 2 \sqrt{P_{\mathrm{p}}} \beta\right)} .
$$

This simple model offers an interesting insight: while single-pass OPA allows us to achieve sub-Poissonian photon statistics in the seed field $(F<1)$, in the presence of a modal mismatch $(\chi<1)$, the degree of intensity squeezing measured by the direct detection does not monotonically grow with the pump power. Instead, the dependence of the sub-Poissonian Fano factor on the pump power shows a turning point, beyond which it asymptotically approaches $F \rightarrow 1$.

The model presented here is meant to provide some physical intuition without being fully rigorous. A full account of the parametric deamplification of a pulsed coherent beam with a finite transverse profile must incorporate a number of phenomena that have not been considered here (e.g., group velocity mismatch between the pump and seed pulses, beam divergence, spatial walk-off, or spatiotemporal coupling in the parametric gain). This will be the subject of a future publication.

Our experimental setup can be divided into two parts: an intensity-squeezing setup and an imaging apparatus (see Fig. 2). Our sub-Poissonian light source is implemented in a single-pass degenerate OPA process, where the pump and seed fields are provided by a

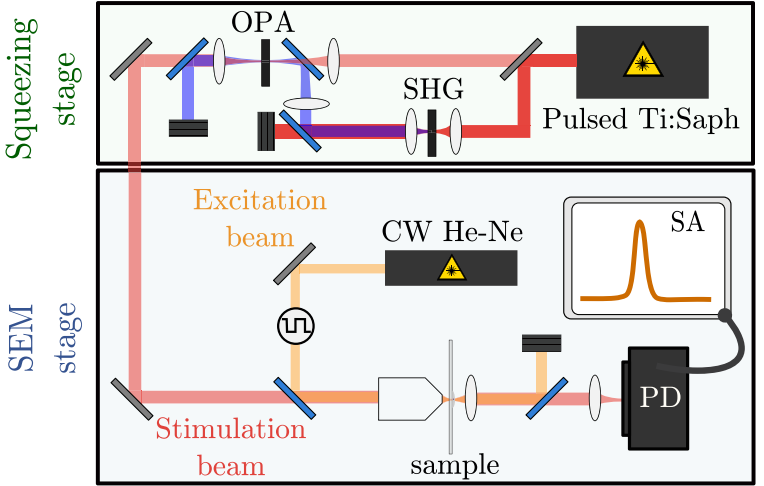

FIG. 2. Experimental setup for the intensity-squeezed light generation (top) and SEM stage (bottom). PD: photodiode; SA: spectrum analyzer.

femtosecond Ti:sapphire laser with a repetition rate of $80 \mathrm{MHz}$, a central wavelength of $\lambda_{\mathrm{S}}=820 \mathrm{~nm}$, a pulse duration of $180 \mathrm{fs}$, and a maximum power of $2.8 \mathrm{~W}$. The fundamental Ti:Saph is spatially filtered and split into strong and weak beams, where the first is frequencydoubled in a second-harmonic generation (SHG) setup (0.4 mm-long type-I $\mathrm{BiBO}$ ) and the latter will act as the seed beam in the OPA. After temporally and spatially overlapping them, the high-intensity pump and low-power seed interact inside a $0.3 \mathrm{~mm}$-long, degenerate, type-I $\mathrm{BBO}$ optical parametric amplifier, after which the pump is filtered out using a low-loss dichroic mirror. This leads to the amplification/deamplification of the seed depending on its phase relative to the pump. We set this phase to yield the maximum deamplification and squeezing using a piezo-controlled mirror in a feedback control loop configuration. The resulting field has a similar pulse shape as the incoming seed while exhibiting reduced intensity fluctuations. ${ }^{17}$ To implement the feedback, the phase of the signal field is very weakly dithered, yielding an error signal proportional to the phase difference between the pump and seed fields when mixed down at the sideband frequency. We verified that this phase modulation of the seed did not introduce a significant mixing of the noise in its squeezed and antisqueezed quadratures.

In a first characterization step, this signal can be directly sent to a photodetector in order to evaluate the degree of intensity squeezing. We employ a custom-made photodetector that achieves a shot-noiselimited direct detection of the deamplified seed in the RF sideband where we place the SEM signal $(\sim 4 \mathrm{MHz})$. Our detector employs a Hamamatsu S5971 silicon PIN photodiode with an 85\% quantum efficiency at $820 \mathrm{~nm}$ and has a bandwidth of $100 \mathrm{MHz}$ when reversebiased at $30 \mathrm{~V}$. The photocurrent is measured in the frequency domain using a high dynamic range signal analyzer.

The SEM imaging stage involves two fields, the excitation beam and the sub-Poissonian stimulation beam, which interact inside the imaged sample. The stimulation beam is prepared in the intensitysqueezing stage described above. The excitation beam is provided by a continuous-wave (CW) He-Ne laser with $\lambda_{\mathrm{E}}=632.8 \mathrm{~nm}$. This field is intensity-modulated with an acousto-optical modulator in order to move the stimulated emission gain to an RF sideband at $4 \mathrm{MHz}$. Note that, ideally, one would excite the sample with ultrafast pulses to maximize the field intensity while minimizing the average power, allow for temporal suppression of additional processes, and avoid deleterious nonlinear effects. ${ }^{4}$ Here, we assume that these considerations can be 
(a)

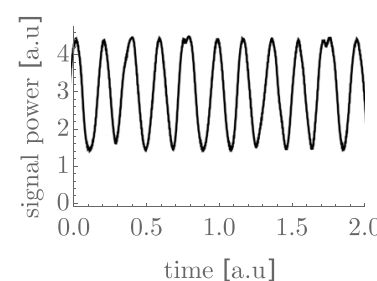

(b)

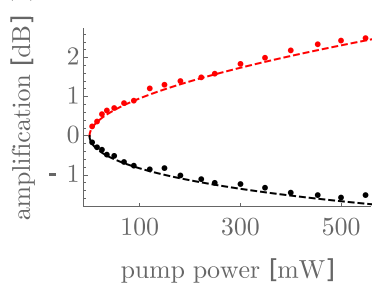

FIG. 3. (a) Amplification/deamplification of the signal as its phase is linearly scanned in time. (b) Maximum amplification (red dots) and deamplification (black dots) as a function of the pump power. The fitted curves (dashed lines) take into account the imperfect mode overlap between the pump and the signal [Eq. (2)].

neglected and use a modulated CW laser with low enough intensity not to saturate the absorption transition, so that repumping takes place on a timescale much longer than the probe pulse duration. The imaged sample is a diluted triphenyl methane (TMP) dye, Brilliant Blue FCF, with a strong absorption band near $\lambda_{\mathrm{E}}$, which is well separated from the spectrum of the stimulation beam. The probe is sufficiently weak to ensure that it does not saturate the gain, even though the relaxation time of the ground state is likely longer than the probe pulse duration. ${ }^{18}$ The excitation and stimulation beams are combined on a dichroic mirror and focused onto the sample using an objective with a numerical aperture of 0.4 , achieving a beam waist of approximately $50 \mu \mathrm{m}$. After the interaction, the excitation beam is filtered out from the stimulation beam by means of two spectral filters. The intensity in the stimulation beam is measured with the photodetector + RF spectrum analyzer arrangement described above.

Let us begin by reporting the performance of our sub-Poissonian light source in the absence of the SEM imaging stage. The degree of intensity squeezing achieved in a single-pass OPA is correlated with the amount of the classical intensity deamplification (see the supplementary material). Figure 3(a) shows the average power of the output signal as the relative phase between the pump and the seed is scanned. A fit of our measurements to the simple model presented in Eq. (2) is shown in Fig. 3(b), where the best fit is obtained for a mode overlap parameter $\chi=0.58$.

The intensity fluctuations of the deamplified seed are expected to be smaller than those of a coherent field of the same average power. Figure 4(a) shows the noise power of our deamplified seed in the frequency range of interest (red). This is compared with the noise power (a)

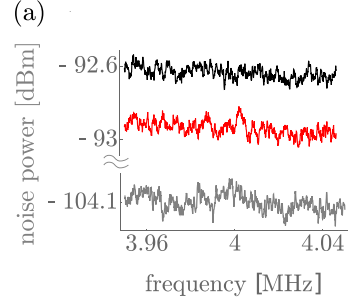

(b)

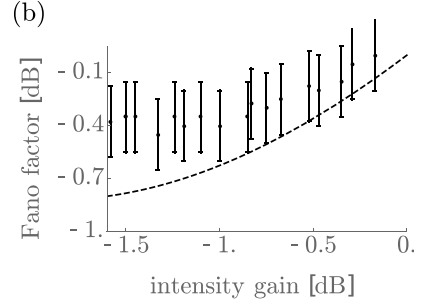

FIG. 4. (a) Noise power of the classical (black) and deamplified seed (red) beams of matching mean intensity and electronic noise of the SA (gray). The resolution bandwidth is $1 \mathrm{kHz}$, and the video bandwidth is $10 \mathrm{~Hz}$. b) Fano factor vs mean intensity deamplification for different pump powers. The dashed line is a fit to Eq. (3). of the same field in the absence of parametric deamplification (black). The average power of the amplified and non-amplified fields is matched using a waveplate + polarizer power control stage. The electronic noise (gray) is almost an order of magnitude below the shot noise, and we have verified that, beyond an analysis frequency of $2 \mathrm{MHz}$, the shot noise exhibits the flat frequency dependence characteristic of quantum fluctuations. The gap between the noise power of the deamplified and non-deamplified seeds clearly signals a Fano factor $F<1$ of the former, proving its sub-Poissonian statistics.

The degree of intensity squeezing is expected to increase with the pump power, similarly to the amount of the classical intensity deamplification. In Fig. 4(b), the Fano factor of the deamplified seed is plotted as a function of the classical intensity deamplification. In contrast to the classical intensity deamplification, the amount of intensity squeezing approaches a plateau. The discrepancy between the squeezing and classical deamplification can be attributed to propagation losses, inefficient detection, and an imperfect mode overlap between the pump and seed beams (e.g., spatial or temporal). We determine the propagation efficiency to be $\eta_{\mathrm{p}}=0.85$ and the detector efficiency to be $\eta_{\mathrm{d}}=0.85$. Using the effective nonlinear parameter and mode overlap obtained in the previous classical deamplification fit, and adding the effect of propagation loss and detection inefficiency, we compute the expected Fano factor using Eq. (3). While this calculation fits the experimental data for the low values of the classical deamplification, it breaks down at higher gains. To understand this discrepancy, a more accurate model of OPA of a pulsed coherent beam is needed. ${ }^{17}$

We now proceed to evaluate the performance of our squeezingenhanced SEM setup. First, we quantify the degradation of squeezing due to the non-ideal transmission efficiency (75\%) of the microscope objective and the dye. As can be seen in Fig. 5, a residual squeezing of $0.3 \mathrm{~dB}$ is measured. Next, we assess the impact of this quantum enhancement on the SEM measurement. The light-red and gray traces in Fig. 5 correspond to the measured noise power for squeezed and classical probes in the presence of an excitation beam that allows the stimulated emission to occur. The average power of the stimulation and excitation beams is set, respectively, to $2 \mathrm{~mW}$ and $30 \mu \mathrm{W}$ to avoid excessive photobleaching. ${ }^{19}$ The appearance of a spectral peak at the frequency of modulation of the excitation beam, and its absence when the stimulation beam is blocked, signals the presence of the stimulated

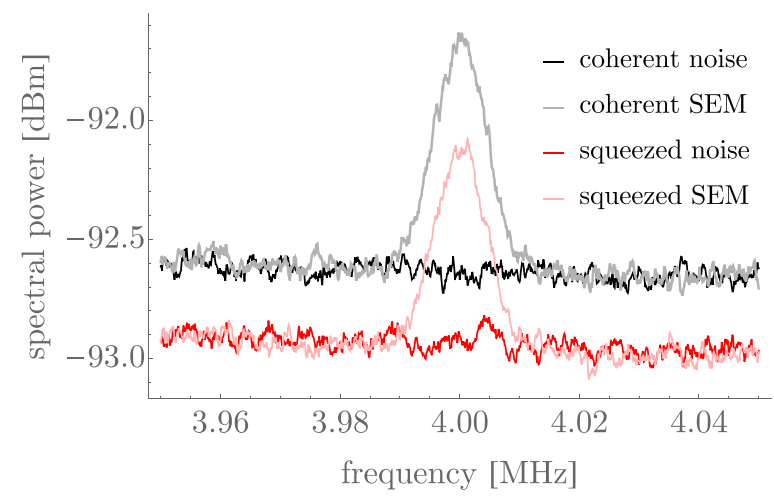

FIG. 5. Stimulated emission microscopy signals for an intensity-squeezed probe (light red) and coherent beam of the same average power (gray). The shot-noise limit is shown in black and the noise power of the squeezed probe is shown in red. 
emission, as well as the absence of the spontaneous emission. As is evident, the shot noise is reduced, and the signal is of comparable magnitude, which signals an improvement in the signal to noise ratio. However, residual photobleaching caused the SEM signal to decay over the measurement time, which made the direct comparison of measurements using squeezed and coherent probes unreliable. Nevertheless, the SNR enhancement, achievable with this technique, can be estimated by assuming that the SEM signal remains constant over the measurements, as suggested by the similar integrated signal powers at the modulation sideband. Under this assumption, Eq. (1) gives the fractional improvement as $\mathrm{SNR}_{\text {squeezed }} / \mathrm{SNR}_{\text {coherent }}$ $=\left(1+\left(G_{0}-1\right)^{2} \rho_{t} /\left(16 G_{0}\right)\right) /\left(F+\left(G_{0}-1\right)^{2} \rho_{t} /\left(16 G_{0}\right)\right)$. This is always larger than unity for $F<1$, no matter how much technical noise is present. The quantitative improvement of the course depends on the latter, and in the present case, this can be estimated. We estimate the sideband stimulated emission gain $\left(G_{0}-1\right)$ to be smaller than $1 \%$, on the grounds that a greater amplification would have been resolved by our photodetection setup in the absence of gain modulation. The value of $\rho_{t}$ is harder to gauge and is not simple to measure directly. A reasonable commercial laser would have a ratio of $\rho_{t}<1$. Allowing for additional noise due to, for example, mode overlap fluctuations, the sample motion and photobleaching would increase this, but it would not be excessive to put an upper bound of $\rho_{t} \sim 1$. This implies that the contribution of the technical noise to the SNR is minor. Therefore, the observed reduction of the shot noise provides evidence that, according to the theoretical model of SEM that we have presented, the average SNR of the measurement was improved by at most $0.3 \mathrm{~dB}$.

In summary, we have proposed and implemented a quantumenhanced nonlinear microscopy technique that employs the stimulated emission to remove the requirement for labeling biological samples. We have identified that shot-noise fluctuations in the stimulation beam currently pose a real limitation in the sensitivity of SEM and have reduced these by preparing an ultrafast probe beam in an intensity-squeezed state. Using a single-pass degenerate OPA, we have achieved the direct observation of the macroscopic light with a Fano factor of $-0.4 \mathrm{~dB}$. Further noise reduction has been prevented by the spatiotemporal mode mismatch and mode mixing in the OPA pump and seed pulses, as well as by the imperfect detection efficiency. We have used this non-classical source of light in conjunction with an SEM imaging setup, observing that sub-Poissonian statistics persist in the measurement signal. Following the theoretical model of SEM that we have developed, this reduction of the shot noise implies an improvement of the SNR of the measurement.

See the supplementary material for our model of signal and noise in stimulated emission measurements, as well as of intensity squeezing in the parametric amplification of coherent states.

This project was supported by the Engineering and Physical Sciences Research Council (EPSRC) Quantum Technology Hub in Quantum Enhanced Imaging (QuantIC) No. EP/M01326X/1. G.T.G. thanks Merton College, Oxford, for its support.

We thank N. Treps and J. Francis-Jones for their fruitful discussions.

\section{DATA AVAILABILITY}

The data that support the findings of this study are available from the corresponding author upon reasonable request.

\section{REFERENCES}

${ }^{1}$ S. W. Hell and J. Wichmann, Opt. Lett. 19, 780 (1994).

${ }^{2}$ S. T. Hess, T. P. Girirajan, and M. D. Mason, Biophys J. 91, 4258 (2006).

${ }^{3}$ M. J. Rust, M. Bates, and X. Zhuang, Nat. Methods 3, 793-795 (2006).

${ }^{4}$ W. Min, S. Lu, S. Chong, R. Roy, G. R. Holtom, and X. S. Xie, Nature 461, 1105 (2009).

${ }^{5}$ C. Cremer and U. Birk, Front. Phys. 4, 11 (2016).

${ }^{6}$ T. Ono, R. Okamoto, and S. Takeuchi, Nat. Commun. 4, 2426 (2013).

${ }^{7}$ N. Samantaray, I. Ruo-Berchera, A. Meda, and M. Genovese, Light: Sci. Appl, 6, e17005 (2017).

${ }^{8}$ P. Moreau, E. Toninelli, T. Gregory, and M. J. Padgett, Nat. Rev. Phys. 1, 367 (2019).

${ }^{9}$ R. Tenne, U. Rossman, B. Rephael, Y. Israel, A. Krupinski-Ptaszek, R Lapkiewicz, Y. Silberberg, and D. Oron, Nat. Photonics 13, 116 (2019).

${ }^{10}$ M. Taylor, J. Janousek, V. Daria, J. Knittel, B. Hage, H.-A. Bachor, and W. Bowen, Nat. Photonics 7, 229-233 (2012).

${ }^{11}$ R. B. de Andrade, H. Kerdoncuff, K. Berg-Sørensen, T. Gehring, M. Lassen, and U. L. Andersen, Optica 7, 470 (2020).

${ }^{12}$ C. A. Casacio, L. S. Madsen, A. Terrasson, M. Waleed, K. Barnscheidt, B. Hage, M. A. Taylor, and W. P. Bowen, arXiv:2004.00178 (2020).

${ }^{13}$ D. F. Walls, Nature 306, 141 (1983).

${ }^{14}$ V. Sundstrom, T. Gillbro, and H. Bergstrom, Chem. Phys. 73, 439 (1982).

${ }^{15}$ E. N. Ivanov, S. A. Diddams, and L. Hollberg, IEEE Trans. Ultrason., Ferroelectr., Freq. Control 50, 355 (2003).

${ }^{16}$ R. Schnabel, Phys. Rep. 684, 1 (2017).

${ }^{17} \mathrm{G}$. Triginer, V. Thiel, and I. Walmsley, "Design of optimized sources of pulsed sub-Poissonian light" (unpublished).

${ }^{18}$ R. Marsh, D. Armoogum, and A. Bain, Chem. Phys. Lett. 366, 398 (2002).

${ }^{19}$ L. Song, E. J. Hennink, I. T. Young, and H. J. Tanke, Biophys. J. 68, 2588 (1995). 